\title{
Anatomical changes in the East Asian midface skeleton with aging
}

\author{
A. Jeon ${ }^{1}$, K.H. Sung ${ }^{1}$, S.D. Kim², U.-Y. Lee ${ }^{3}$, J.-H. Lee ${ }^{4}$, S.-H. Han' ${ }^{1}$, H.-J. Sui ${ }^{5}$ \\ ${ }^{1}$ Department of Anatomy, College of Medicine, Chung-Ang University, Seoul, Korea \\ ${ }^{2}$ Bright and Clear Dermatology Clinic, Seoul, Korea \\ ${ }^{3}$ Department of Anatomy, Catholic Institute for Applied Anatomy, College of Medicine, \\ The Catholic University of Korea, Seoul, Korea \\ ${ }^{4}$ Anatomy Laboratory, College of Sports Science, Korea National Sport University, Seoul, Korea \\ ${ }^{5}$ Department of Anatomy, College of Basic Medicine, Dalian Medical University, Dalian, China
}

[Received: 6 February 2017; Accepted: 14 March 2017]

Background: Understanding the aging process of the midface skeleton is considered crucial for correct facial rejuvenation. However, the canine fossa, an important morphological feature of the midface skeleton, has not yet been observed in connection with aging, despite the fact that it is the most main part of the maxillary bone. Here, the authors focus on the depression of the canine fossa to evaluate the Asian midface skeleton.

Materials and methods: Computed tomography (CT) scans of the facial skeleton of 114 Koreans (59 males and 55 females) were reconstructed to three-dimensional (3D) images using a 3D analysis software programme. The study subjects included 27 young males, 32 old males, 28 young females and 27 old females. The angular measurements of three bony regions were measured for each 3D model: the canine fossa angle (assessing depth of the canine fossa), the maxillary angle (assessing orientation of the lateral maxilla) and the piriform angle (assessing orientation of the medial maxilla).

Results: The canine fossa angle showed a statistically significant decrease with aging in both sexes, indicating the canine fossa actually becomes more concave with age. In contrast, the maxillary and piriform angle showed statistically insignificant changes with aging in female subjects.

Conclusions: These results suggest that the canine fossa may be one of the effective markers to evaluate the anatomical changes to the facial skeleton with midface aging. (Folia Morphol 2017; 76, 4: 730-735)

Key words: aging changes, facial skeleton, three-dimensional study, canine fossa, maxilla, piriform aperture

\section{INTRODUCTION}

The aging process of face can be summarised into two distinct features: gravitational sagging and volume loss. Within this notion, numerous manoeuvres are being developed to regain the youthfulness of the face, whether to lift the face up with various lifting procedures, or to restore its volume in a 3-dimensional (3D) manner. Cosmetic surgeons understand that the mechanism of facial aging is a complex process and involves various anatomical layers of face, from epidermal atrophy producing folds to bone desorption resulting in the multi-faceted contours of an 
aged face. Among the various treatment modalities, mid-face volume enhancement is regarded as one of the most powerful tools to restore youthfulness to the face, as the midface skeleton undergoes the most dramatic changes with aging. From an osteological point of view, the mid-facial bones, which are the thinnest part in the human skull [17], might be vulnerable to bone desorption with aging.

The aging process of the bone, also known as bone remodelling, occurs throughout the lifetime of every human being $[12,19]$. This metabolism is caused by bone resorption and the formation to balance homeostasis. The activity of the bone remodelling process increases bone volume during the growth period, and later decreases, resulting in loss of bone volume with aging. The facial skeleton also undergoes bone resorption after the growth period.

The canine fossa underlies the nasolabial fold, which is one of the main characteristics of interpreting faces; furthermore, it is the most unique marker of aging faces. We speculate that the canine fossa would exhibit changes with age following an identical pattern to other parts of the facial skeleton. However, the canine fossa was excluded from the measurement list of previous investigations to assess midface skeleton aging. In this study, we focused on changes to the canine fossa in relation to midface aging. Lambros suggested that the facial skeleton continued remodelling in a clockwise rotation of the midface with aging of Caucasian. Moreover, Lambros's theory has been underpinned by Pessa [13], and further confirmed by Shaw and Kahn [17]. In spite of this, the mechanism of facial skeletal aging has not yet been fully clarified in Asians.

The present study was performed to evaluate the aging process of the Asian midface skeleton in detail.

\section{MATERIALS AND METHODS}

Data was collected from computed tomography (CT) scans of the facial skeleton, at a slice width of $0.60 \mathrm{~mm}$ using a 64-channel multidetector CT (SOMATOM Sensation 64, Siemens, Forchheim, Germany). CT scans were reconstructed to $3 \mathrm{D}$ images using the 3D analysis software programme called Mimics ${ }^{\mathrm{TM}}$ (Materialise NV, and Belgium). Subjects who exhibited any signs of previous facial fractures and craniofacial deformities were excluded [14].

A total of 114 Koreans (59 males and 55 females) were enrolled in this study, all of whom reside in South Korea. Demographic data were further ana- lysed for the 114 subjects according to their sex and age: 27 young males (22-35 years), 32 old males (60-80 years), 28 young females ( $21-35$ years), and 27 old females (60-83 years). This study was approved by the IRB of Chung-Ang University (IRB No: 1041078-201411-HR-185-01).

In order to evaluate the angular changes with aging, angular measurements of three bony regions (the canine fossa, maxilla and piriform aperture) were made using a method based on 3D vector mathematics. A line from the sella to the nasion was taken as a reference. The measurement points of the maxilla and piriform aperture were selected in accordance with Pessa's work [13] in order to allow for a comparison with other population groups, especially Caucasians. The canine fossa could be defined as a bony depression on the anterior maxillary surface below the infraorbital foramen, which resides on the lateral side of the canine eminence, also known as the maxillary fossa.

All measurements were taken in degrees. Measurements were conducted on the left side. The canine fossa angle was defined as an angle formed by a line traversing the uppermost joint point at the zygomaxillary suture to the maximum maxillary curvature (deepest point between the upper alveolar process and zygomaxillary suture) [2] and a line formed by sella-nasion line. Changes to the maxillary angle (superior to inferior maxilla at the joint of the inferior maxillary wing and alveolar arch) and the piriform angle (from the nasal bone to the lateral inferior piriform aperture) were measured according to Pessa's work (Fig. 1).

\section{Statistical analysis}

All measurements were obtained using an identical software programme, Mimics ${ }^{\mathrm{TM}}$, in degrees. This programme creates and edits the measurement points and planes. Data was analysed with a Student's t-test to identify any trends between young and old groups using SPSS version 21.0 (IBM SPSS Inc., Chicago, IL, USA), with a value of $p<0.05$ considered statistically significant. The intraclass correlation coefficient was used to examine the intraobserver reproducibility of measurement scale (at a confidence level of $95 \%$ ).

\section{RESULTS}

The mean age in the young group was 27.3 years for male subjects and 28.2 years for female subjects. In the old group, the mean age was 64.9 years for the male subjects and 66.2 years for the female subjects 


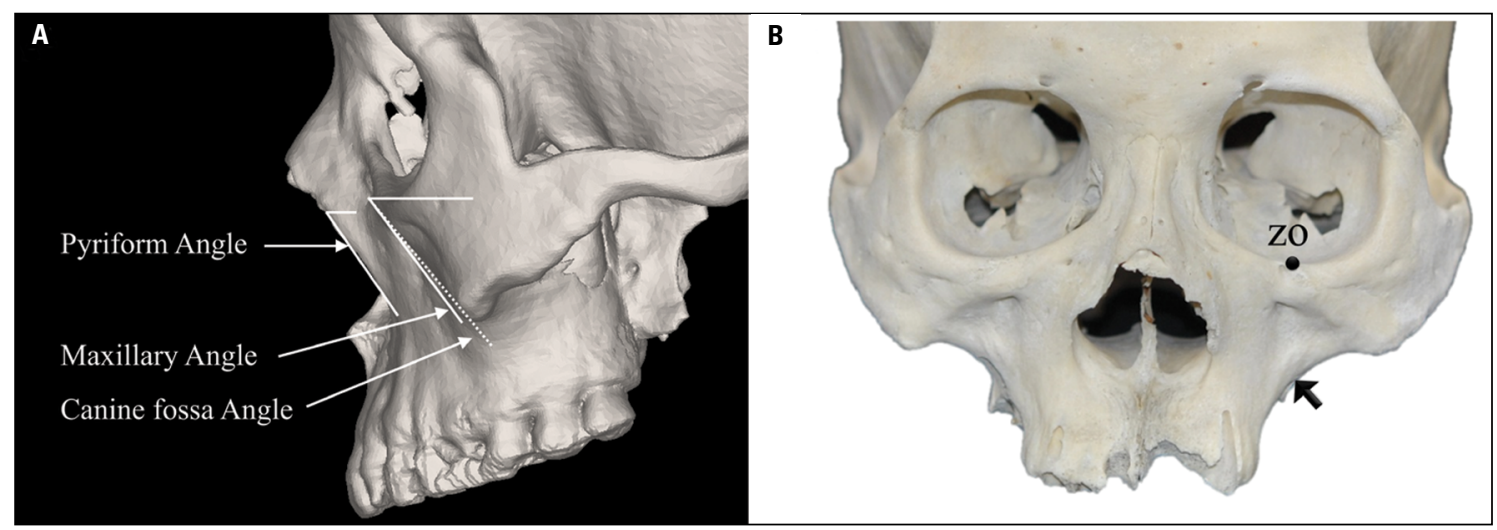

Figure 1. A. Sample of 3-dimensional computed tomography of a younger male subject with the three angular measurements applied; B. Picture depicting the reference points of the canine fossa. The arrow indicates the deepest point between the upper alveolar process and zygomaxillary suture; zo — zygoorbitale.

Table 1. Average age by sex in each age category

\begin{tabular}{lccccc}
\hline Age & \multicolumn{3}{c}{ Young } & & \multicolumn{2}{c}{ Old } \\
\cline { 2 - 3 } \cline { 5 - 6 } & Male $(\mathbf{n}=\mathbf{2 7})$ & Female $(\mathbf{n}=\mathbf{2 8})$ & & Male $(\mathbf{n}=\mathbf{3 2})$ & Female $(\mathbf{n}=\mathbf{2 7})$ \\
\hline Mean \pm SD & $27.3 \pm 4.0$ & $28.2 \pm 4.4$ & & $64.9 \pm 4.3$ & $66.2 \pm 5.0$ \\
Range & $22-35$ & $21-35$ & & $60-80$ & $60-83$ \\
\hline
\end{tabular}

SD - standard deviation

Table 2. Angular measurement of three facial areas by sex

\begin{tabular}{|c|c|c|c|c|c|}
\hline \multirow[t]{2}{*}{ Measurement } & \multicolumn{2}{|c|}{ Age category } & \multirow[t]{2}{*}{ Difference } & \multirow[t]{2}{*}{$\mathbf{P}$} & \multirow{2}{*}{$\begin{array}{l}\text { Intraclass correlation coefficients } \\
\text { (95\% confidence interval) }{ }^{\#}\end{array}$} \\
\hline & Young & Old & & & \\
\hline \multicolumn{6}{|c|}{ Canine fossa angle $\left({ }^{\circ}\right)$} \\
\hline Male & $56.6 \pm 3.9$ & $53.3 \pm 4.2$ & 3.29 & $0.0050^{\dagger}$ & 0.990 \\
\hline Female & $54.7 \pm 4.0$ & $50.6 \pm 4.5$ & 4.17 & $0.0010^{\dagger}$ & 0.987 \\
\hline \multicolumn{6}{|c|}{ Maxillary angle $\left({ }^{\circ}\right)$} \\
\hline Male & $53.9 \pm 3.3$ & $51.0 \pm 4.6$ & 2.99 & $0.0176^{\dagger}$ & 0.990 \\
\hline Female & $52.8 \pm 4.5$ & $50.6 \pm 4.3$ & 2.15 & 0.0860 & 0.988 \\
\hline \multicolumn{6}{|c|}{ Piriform angle $\left({ }^{\circ}\right)$} \\
\hline Male & $59.6 \pm 4.9$ & $56.3 \pm 3.6$ & 3.30 & $0.0075^{\dagger}$ & 0.975 \\
\hline Female & $59.8 \pm 4.5$ & $58.1 \pm 5.3$ & 1.69 & 0.2160 & 0.973 \\
\hline
\end{tabular}

Mean measurements shown in degrees \pm standard deviation; ${ }^{\dagger}$ Statistically significant change $(p<0.05)$; ${ }^{\# T h e ~ i n t r a c l a s s ~ c o r r e l a t i o n ~ c o e f f i c i e n t s ~ o f ~ t h e ~ r e p e a t e d ~ m e a s u r e m e n t s ~}$ (at a confidence level of 95\%).

(Table 1). The results of the three angular measurements are shown in Table 2 and Figure 2.

\section{Canine fossa angle}

The canine fossa angle showed a marked change with aging. There were statistically significant changes in both male $\left(56.6^{\circ}\right.$ young vs. $53.3^{\circ}$ old, $p=0.0050$ ) and female $\left(54.7^{\circ}\right.$ young vs. $50.6^{\circ}$ old, $\left.p=0.0010\right)$ subjects. The mean canine fossa angles in the male and female subjects were decreased by $3.3^{\circ}$ and $4.1^{\circ}$ with aging, respectively.

\section{Maxillary angle}

The mean maxillary angle in the male subjects was significantly decreased by $2.9^{\circ}$ with aging $\left(53.9^{\circ}\right.$ young vs. $51.0^{\circ}$ old, $\left.p=0.0176\right)$. In the female 

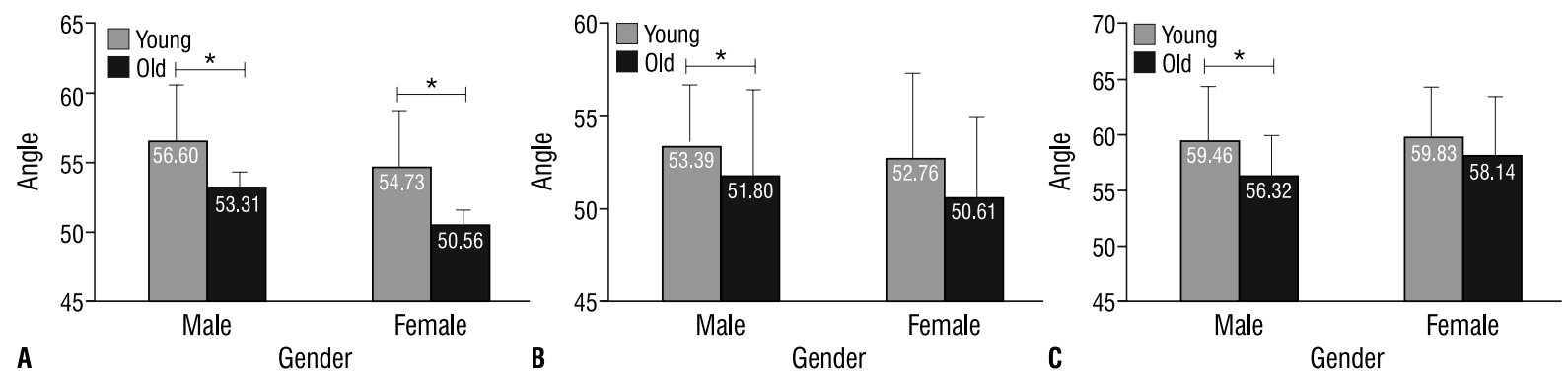

Figure 2. Column graphs illustrating the three angular measurements. A. Canine fossa angle; B. Maxillary angle; C. Pyriform angle. Statistically significant decreases were observed in the canine fossa angle with aging for both sexes. Maxillary and piriform angle showed statistically insignificant changes in female subjects; ${ }^{*} p<0.05$.

subjects, the mean maxillary angle was decreased by $2.2^{\circ}$ with aging, but it was not statistically significant (52.8 $8^{\circ}$ young vs. $50.6^{\circ}$ old $)$.

\section{Piriform angle}

The mean angle in the male subjects was significantly decreased by $3.3^{\circ}$ with aging $\left(59.6^{\circ}\right.$ young vs. $56.3^{\circ}$ old, $p=0.0075$ ). In the female subjects, the change with aging was not statistically significant (59.8 $8^{\circ}$ young vs. $58.1^{\circ}$ old $)$.

\section{DISCUSSION}

The maxillary angle, which has been suggested as an indicator for mid skeletal aging in previous studies, may incompletely interpret the degree of mid skeletal aging, as it designates a reference point close to the zygomatic bone. To overcome this inaccuracy, the canine fossa was measured in this study as the fundamental part of the maxillary bone and the deepest part in the midface skeleton. Our results confirmed that the canine fossa becomes more concave with increasing age.

Previous studies have supported Lambros's theory, which described the facial changes with age of the midface skeleton on the right side aspect as a clockwise rotation of the maxilla relative to the cranial base $[4,9,13,15,17,18]$. Pessa in 2000 [13], studied how the midface skeleton changes with increasing age in the young and old groups. These measurements were taken from 3D stereolithographic images from 12 male subjects. Pessa [13] reported noticeable changes in the maxilla and piriform angles with increasing age, and the mean values of the differences were $13.8^{\circ}$ and $10.7^{\circ}$, respectively. Shaw and Kahn, in 2007 [17], also studied the changes of the midface skeleton using 3D tomography. They similarly noted a significant decrease in the maxillary angle when comparing young and old age groups, but no noticeable change in the piriform angle was observed. Another study, by Shaw et al. in 2011 [18], was performed on a larger scale of 120 Caucasian subjects. The decrease in the maxillary angle exhibited a similar pattern to their study in 2007. Mendelson et al. [8] confirmed the reduction of the maxillary angle with aging. Compared with the above-mentioned previous studies, our data exhibited a far lower decrement of angle of $2.9^{\circ}$ between younger and older male subjects. This significant difference of decrement could be explained by different morphology between Asian and Caucasian skulls. Asians tend to possess a higher arched zygomatic bone $[5,11]$. This implies that the maxillary angle would not only reflect the degree of midface skeletal resorption, but also the degree of zygoma desorption.

In this study, the piriform aperture showed statistically insignificant change with aging only in female subjects, exhibiting discrepancy with the results of Shaw and Kahn [17], concluding that the piriform angle showed statistically insignificant change in both sexes. This discrepancy might be explained by diverse morphologic features of the nasal bone among human beings $[3,6]$, which led to bias in interpreting the midface skeleton aging. The diverse pattern of the nasal bone might further explain the discrepancy of between the results of Shaw and Kahn [17] and those of Pessa [13] in Caucasian groups, concluding that the piriform angle showed marked reduction of the piriform angle. In conclusion, our study might infer that the piriform angle might not be a useful marker to evaluate midface skeleton aging.

When evaluating midface skeleton aging, various factors must be considered: ethnic differences of the facial skeleton and selection of right reference points to avoid possible biases induced by different individual morphological characteristics. Several 


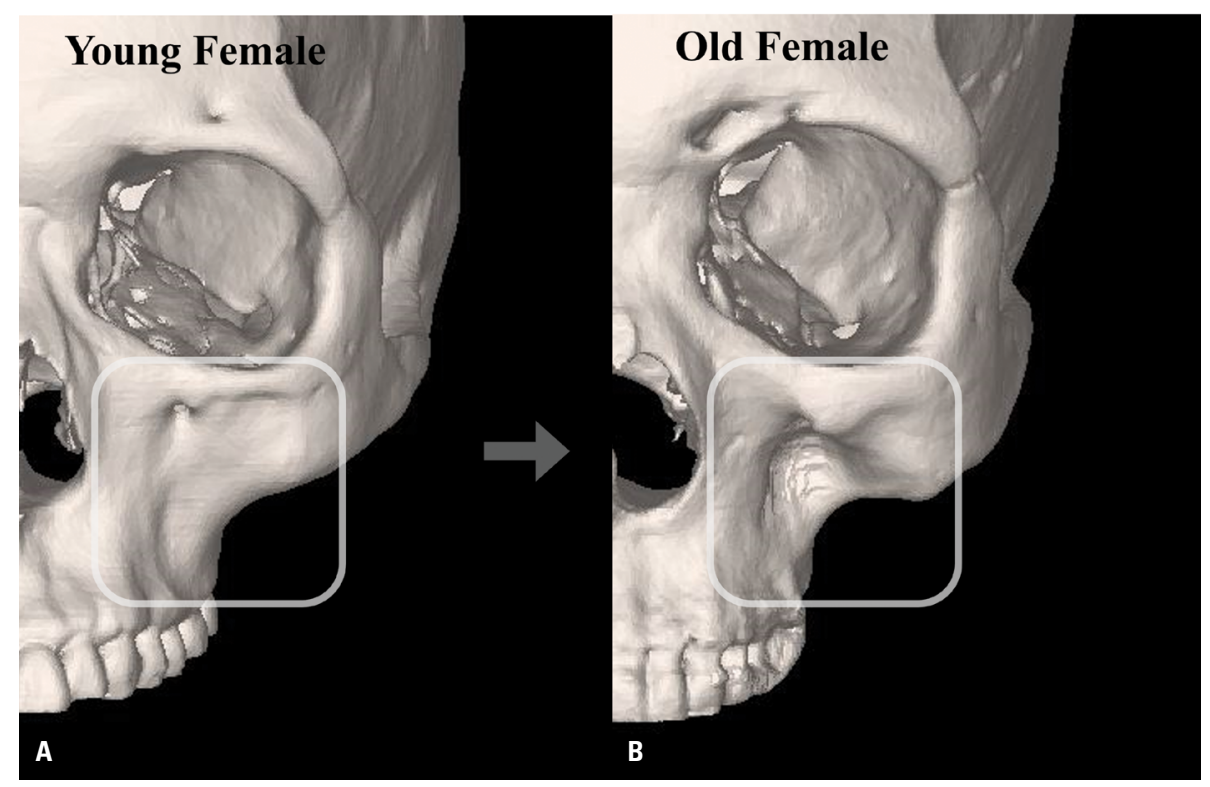

Figure 3. A 3-dimensional reconstruction of the canine fossa of a young female subject (A) and an old female subject (B). A marked retrusion was observed in the canine fossa with aging.

studies indicate that there are significant differences between Asian skulls and Caucasian skulls [1, 10]. Moreover, racial differences of bone thickness and density may affect discrepancy of the results in designing a study [16].

Kim et al. [4] reported that the maxillary angle in both sexes and the piriform angle in females showed a significant decrease with aging. They suggested that any facial skeleton changes in Asians showed clockwise rotation around the orbit with aging, which coincided with previous studies. In contrast, our present study found that Asian facial skeletons do not follow the typical aging patterns proposed by Lambros. The canine fossa angle was the only decreasing angle in both sexes among three angular measurements as age increases. The decrease of the maxillary angle showed minimal changes, compared to that of Caucasians $[8,13,15,17,18]$.

Compared with the study conducted by Kim et al. [4] on an Asian population, the results of our study have revealed a different pattern in midface skeleton aging. This discrepancy may be explained by the different methodological approach utilised in our study. Kim et al. [4] measured the angles on CT images and calculated measurements using 3D vectors, while in our study, 3D analysis was undertaken, the method which was selected by Shaw and Kahn [17]. The difference in measurement method utilised may have influenced the results.
Our present study demonstrated that the canine fossa changed with aging in older groups (Fig. 3), regardless of sex, compared with changes of the maxilla and piriform aperture. Moreover, it showed meaningful changes in both sexes (Table 2, Fig. 2). However, previous studies focused on the maxilla as the key player in midface aging $[4,8,9,15,17,18]$. The canine fossa is an unfamiliar marker in midface skeletal aging. Lee et al. [7] utilised the degree of concavity of the canine fossa to merely classify morphological characters of the infraorbital foramen, which had no relevance with aging. To our knowledge, this study is the first attempt to utilise the canine fossa as a marker for midface skeleton aging. The canine fossa may be a suitable candidate in midface aging as numerous facial muscles and fat pads overlies the canine fossa, of which its retrusion may contribute to an aging face. Evaluating and comparing the canine fossa might be a valuable marker to elucidate the degree of maxillary retrusion in terms of aging than conventional measurement points, such as the maxillary angle. We suggest the canine fossa as one of the most effective markers to evaluate anatomical changes in the facial skeleton with midface aging.

To improve accuracy, all the measuring points were designed based on 3D vector mathematics. We reconstructed whole facial $\mathrm{CT}$ data into $3 \mathrm{D} \mathrm{im}$ ages to reduce the ambiguity of the measuring on cross-sectional images. Interestingly, the depth of the 
canine fossa exhibited individual variation. Further studies are required to classify the degree of concavity of the canine fossa with aging.

Numerous factors, such as individual, environmental, or biological factors, may affect the aging process of the facial skeleton, regardless of race. To fulfil the goal of rejuvenating Asian patients, appropriate individualisation strategies must be established. Individualised treatment strategies could only be produced by understanding these racial aging differences and anatomical variations between races. It is imperative to recognise and compare different physical appearances between Asians and Caucasians, which may further individualize and establish optimal treatment strategies for the best outcomes in the facial cosmetic field.

\section{CONCLUSIONS}

These results suggest that the canine fossa may be one of the effective markers to evaluate the anatomical changes to the facial skeleton with midface aging.

\section{Acknowledgements}

This research was supported by Basic Science Research Programme through the National Research Foundation of Korea (NRF) funded by the Ministry of Science, ICT and Future Planning (2017R1A2B4005787). This work was supported by Health Fellowship Foundation.

\section{REFERENCES}

1. Gu Y, McNamara JA, Sigler LM, et al. Comparison of craniofacial characteristics of typical Chinese and Caucasian young adults. Eur J Orthod. 2011; 33(2): 205-211, doi: 10.1093/ejo/cjq054, indexed in Pubmed: 20709723.

2. Howells WW. Cranial variation in man. A study by multivariate analysis of patterns of difference. Among recent human populations. Papers of the Peabody Museum of Archaeology and Ethnology. 1973(67): 1-259.

3. Hwang TS, Song J, Yoon Ho, et al. Morphometry of the nasal bones and piriform apertures in Koreans. Ann Anat. 2005; 187(4): 411-414, doi: 10.1016/j.aanat.2005.04.009, indexed in Pubmed: 16163855.

4. Kim YK, Lee HB. Lift on asian: consideration on third purse string suture and malar region. Journal of the Korean Society for Aesthetic Plastic Surgery. 2009; 15(2): 116-120.

5. Kim SJ, Kim SoJ, Park JS, et al. Analysis of age-related changes in asian facial skeletons using 3D vector mathematics on picture archiving and communication system computed tomography. Yonsei Med J. 2015; 56(5): 1395-1400, doi: 10.3349/ymj.2015.56.5.1395, indexed in Pubmed: 26256986.
6. Lang J, Baumeister R. Über das postnatale Wachstum der Nasenhöhle. Gegenbaurs Morphol Jb. 1982; 128: 354-493.

7. Lee UY, Nam SH, Han SH, et al. Morphological characteristics of the infraorbital foramen and infraorbital canal using three-dimensional models. Surg Radiol Anat. 2006; 28(2): 115-120, doi: 10.1007/s00276-005-0071-y, indexed in Pubmed: 16432643.

8. Mendelson $\mathrm{B}$, Wong $\mathrm{CH}$. Changes in the facial skeleton with aging: implications and clinical applications in facial rejuvenation. Aesthetic Plast Surg. 2012; 36(4): 753-760, doi: 10.1007/s00266-012-9904-3, indexed in Pubmed: 22580543.

9. Mendelson BC, Hartley W, Scott M, et al. Age-related changes of the orbit and midcheek and the implications for facial rejuvenation. Aesthetic Plast Surg. 2007; 31(5): 419-423, doi: 10.1007/s00266-006-0120-x, indexed in Pubmed: 17486402.

10. Morris DE, Moaveni Z, Lo LJ. Aesthetic facial skeletal contouring in the Asian patient. Clin Plast Surg. 2007; 34(3): 547-556, doi: 10.1016/j.cps.2007.05.005, indexed in Pubmed: 17692710.

11. Neligan P, Warren RJ. Plastic Surgery-Aesthetic. 3rd ed. Vol. 2. Elsevier Health Sciences 2012: 177-178.

12. Parfitt A. Morphologic basis of bone-mineral measurements-transient and steady-state effects of treatment in osteoporosis. Miner Electrolyte Metab. 1980; 4: 273$-287$.

13. Pessa JE. An algorithm of facial aging: verification of lambros's theory by three-dimensional stereolithography, with reference to the pathogenesis of midfacial aging, scleral show, and the lateral suborbital trough deformity. Plastic Reconstr Surg. 2000; 106(2): 489-490, doi: 10.1097/00006534-200008000-00041.

14. Prein J. Manual of Internal Fixation in the Cranio-Facial Skeleton: Techniques as recommended by the AO/ASIF-Maxillofacial Group. Springer Science \& Business Media 1998.

15. Richard MJ, Morris C, Deen BF, et al. Analysis of the anatomic changes of the aging facial skeleton using computerassisted tomography. Ophthal Plast Reconstr Surg. 2009; 25(5): 382-386, doi: 10.1097/IOP.0b013e3181 b2f766, indexed in Pubmed: 19966653.

16. Seeman E. Growth in bone mass and size--are racial and gender differences in bone mineral density more apparent than real? J Clin Endocrinol Metab. 1998; 83(5): 1414-1419, doi: 10.1210/jcem.83.5.4844, indexed in Pubmed: 9589631.

17. Shaw RB, Kahn DM. Aging of the midface bony elements: a three-dimensional computed tomographic study. Plast Reconstr Surg. 2007; 119(2): 675-81; discussion 682, doi: 10.1097/01.prs.0000246596.79795.a8, indexed in Pubmed: 17230106.

18. Shaw RB, Katzel EB, Koltz PF, et al. Aging of the facial skeleton: aesthetic implications and rejuvenation strategies. Plast Reconstr Surg. 2011; 127(1): 374-383, doi: 10.1097/ /PRS.0b013e3181f95b2d, indexed in Pubmed: 20871486.

19. Sims NA, Martin TJ. Coupling the activities of bone formation and resorption: a multitude of signals within the basic multicellular unit. Bonekey Rep. 2014; 3: 481, doi: 10.1038/ bonekey.2013.215, indexed in Pubmed: 24466412. 